\title{
Pattern of computed tomography scan findings in children with Wilms' tumor in a tertiary hospital in Lagos, Nigeria
}

\author{
Adegboyega Olukayode A., \\ Osuoji Richard I. ${ }^{\text {, }}$ \\ Akinola Rachael A., \\ Balogun Babajide O., \\ Faturoti Ireti $O{ }^{{ }^{\prime} \text {, }}$ \\ Awosanya Gbolahan $O$.
}

Departments of Radiology and ${ }^{1}$ Surgery, Paediatric Surgery Unit, College of Medicine, Lagos State University Teaching Hospital, Ikeja, Lagos, Nigeria

\begin{abstract}
A B S T R A C T
Context: Abnormal abdominal distension in a child is usually a source of great concern and apprehension in both the parents and the pediatric surgeon. Although Wilms' tumor is the most common malignant abdominal childhood tumor, usually causing abdominal distension, many other less common causes can also result in abdominal distension. Therefore, early diagnosis of Wilms' tumor and distinguishing it from all these other causes is essential. Aims: We intend to document the pattern of computed tomography (CT) scan findings in patients with Wilms' tumor and the peculiarity of its radiological presentation in this environment, highlighting its accuracy and advantages over other imaging modalities. Settings and Design: This is a prospective descriptive study conducted between January, 2011 to January 2012, where all infants, 6-96 months of age, who were suspected to have Wilms' tumor were made to undergo CT scan examination as part of their management work-up. It was carried out in a teaching hospital. Subjects and Methods: The study performed on 12 children age range of 6-96 months old underwent abdominal CT scans as part of their clinical work-up using a GE Hi-speed dual CT scanner . Pre- and post-contrast images were acquired and assessed by the radiologists. They were operated and their radiological and histopathology results were correlated. Statistical Analysis: All data was entered into a Microsoft word excel spread sheet and analyzed by simple analysis. Percentages and proportions were determined for categorical variables. Results: Of the 12 patients seen, nearly $75 \%$ were males, with a male to female ratio of 3:1 and an average age of 37 months. The right sided tumors were slightly more than the left and nearly $25 \%$ were bilateral. Approximately $17 \%$ showed punctuate calcifications. Heterogeneous contrast enhancement was seen in all the cases, vascular encasement in three and lymphadenopathy in six cases. There was no bony involvement in any of them but $75 \%$ of the masses seen, crossed the midline. Histological confirmation of Wilms' tumor was obtained in all cases. Conclusions: Majority of the tumors seen in this study crossed the midline and a few of them showed calcification. CT scan is relevant and accurate in investigating children with Wilms' tumor as it reveals concomitant lesions with involvement of the abdominal vessels and lymph nodes.
\end{abstract}

Key words: Children, computed tomography scan, Nigeria, Wilms' tumor

\section{INTRODUCTION}

Wilms tumor is the most common cause of abdominal tumors in children in Sub-Saharan Africa, ${ }^{[1-5]}$ accounting for about $6 \%$ of all pediatric malignancies in the United States. ${ }^{[3,4]}$ Other less common causes of abdominal

\begin{tabular}{|l|l|}
\hline \multicolumn{3}{|c|}{ Access this article online } \\
\hline Quick Response Code: & Website: \\
\hline & www.jmpo.org \\
\hline
\end{tabular}

childhood tumor include neuroblastoma, multicystic kidney disease, hydronephrosis and polycystic kidneys. Its incidence is 1 in 10,000 children, representing about 650 new cases annually in the United States. ${ }^{[3,4]}$ It tends to occur between ages 1 and 3 years and $90 \%$ of new cases are diagnosed before 7 years. Occasionally, Wilms' tumor has been seen in teenagers and adults.

Clinically, most children have an asymptomatic abdominal mass, ${ }^{[3-5]}$ usually first discovered by the parent while bathing the child or by the pediatrician during routine physical examination. A third of the infants have intermittent abdominal pain that may be exacerbated by abdominal trauma. Gross or microscopic hematuria is found in up to 
$25 \%$ of them, and hypertension may be present due to an increase in renin secretion by the tumor or from displaced kidney or renal artery by tumor growth. ${ }^{[3,4]}$

Sometimes, fever, malaise and anemia occur as a result of tumor necrosis with intraparenchymal bleeding.

Examination usually reveals a large smooth, non-tender, immobile and firm abdominal mass that does not cross the midline. Intravascular extension of the tumor may present with cardiac murmur, hepatosplenomegaly, ascites, varicocele, or gonad metastasis. ${ }^{[3]}$

It is a known fact that modern imaging techniques are valuable for diagnosis of Wilms' tumor before confirmation by histology. ${ }^{[6]}$ Imaging and various radiographic features help to make a distinction of the various renal masses and thus make a precise preoperative diagnosis. Recent advances in high end on imaging techniques have shown that it is possible to diagnose this disease entity in its early stage in developed society, but in resource limited countries such as ours, patients present late, making its management challenging. In such cases, the tumor may be too large and may have metastasized before presentation. ${ }^{[7]}$ Ultrasound with Color Doppler, Computed tomography (CT) scan and magnetic resonance imaging (MRI) are all modern imaging techniques that have proved useful for diagnosis. However, the cross sectional imaging like CT scan and MRI have been shown to be superior to ultrasonography. ${ }^{[2,8]}$ CT has been found to have accuracy of $82 \%$ and capable of an explicit report in $89 \%$ of all cases..$^{[9]}$

Most of the causes of abdominal distension in children can be differentiated from Wilms' tumor using ultrasound scan. ${ }^{[10]}$ Neuroblastoma commonly crosses the midline and usually calcifies. Since it is a suprarenal tumor, it displaces the kidney while Wilms' tumor destroys and distorts the calyces because it is intrarenal. ${ }^{[11]}$

Multicystic kidneys are the most common cause of masses in neonates ${ }^{[10]}$ and it is best differentiated from other abdominal masses such as hydronephrosis, mesonephric nephroma and Wilms' tumor by ultrasound. ${ }^{[10]}$ Ultrasonography demonstrates a large unilateral renal mass with $10-20$ cysts, but sometimes as many as 50 cysts of various sizes. Islands of dysplastic renal tissue may be observed between the cysts, but no normal tissue is seen. ${ }^{[10]}$

In hydronephrosis, the entire collecting system is outlined as a series of connected fluid-filled channels. When only part of the renal collecting system is dilated, the condition may superficially resemble a tumor on sonographic examination, but close scrutiny can easily differentiate the 2 conditions. ${ }^{[10]}$
Although in Polycystic kidneys, ultrasonography shows diffusely enlarged kidneys with a generalized increase in echoes produced by the innumerable fluid-tubular wall interfaces. The renal borders are poorly defined and corticomedullary differentiation is lost. ${ }^{[10]}$

Wilms' tumor is treated by a chemotherapy regimen of vincristine/dactinomycin or vincristine/dactinomycin/ doxorubicin and radical nephrectomy. ${ }^{[3]}$

At 5-7 year survival rate has tremendously improved from $30 \%$ several decades ago to nearly $90 \%$ today. This is attributed to the success of a multidisciplinary approach and the efforts of cooperative study groups which are, the National Wilms' Tumor Study in North America and the International Society of Pediatric Oncology in Europe. ${ }^{[3,4]}$ Today, Wilms' tumor is one of the most successfully treated pediatric solid malignancies. ${ }^{[3,4]}$

We therefore sought to elicit the pattern of CT scan findings in Wilms' tumor, its peculiar presentation in our environment and the usefulness and accuracy of CT. Its advantages over other modes of imaging will also be highlighted.

\section{SUBJECTS AND METHODS}

This is a prospective descriptive study conducted between January, 2011 and January 2012, where all infants, 6-96 months of age, who were suspected to have Wilms' tumor were made to undergo CT scan examination as part of their management work-up. An informed consent was obtained from each parent. The hospital research and Ethics committee approved the conduct of this study and the conduct was in accordance with the Helsinki Declaration of 1975. There were 12 participants in all and they had abdominal CT scans performed using a GE Hi-speed dual CT scanner ${ }^{\circledR}$, (General Electric co. Milwaukee Wisconsin USA, 2006). Contiguous $1 \mathrm{~cm}$ thick slices at $1 \mathrm{~cm}$ intervals were obtained. Pre- and post-contrast series were acquired with intravenous administration of non-ionic contrast medium at a dose of $2 \mathrm{ml} / \mathrm{kg}$ body weight to opacify the vessels and oral contrast to delineate bowel loops. Most of them had manual injection of contrast while a mechanical injector was used for older patients at $1 \mathrm{~mL} / \mathrm{s}$ injection rate. Volume scanning commenced at the end of manual or mechanical injection but in some cases, a 70 min delay from the inception of the injection was necessary.

All data was entered into a Microsoft word excel spread sheet and analyzed 2007. Simple descriptive statistics (minimum, maximum, mean and standard deviation) were calculated for continuous variables. Percentages and proportions were determined for categorical variables. 


\section{RESULTS}

Table 1 shows the CT scan findings. Of the 12 patients seen, nine $(75 \%)$ were males, with a male to female ratio was $3: 1$.

Majority of them were of an average age of 37 months (3 years). All had huge heterogeneously dense abdominal masses causing abdominal distension and their scanogram showed bulging of the flanks and loss of the psoas muscle outline [Figure 1].

There were $33.3 \%$ left sided tumors, $42 \%$ right sided tumors and three bilateral tumors (25\%). Approximately, $17 \%$ tumors showed punctuate or speckle calcifications [Figure 2].

Heterogeneous contrast enhancement was seen in all the cases [Figure 3]. There was vascular encasement in three cases while lymph node enlargement was seen in six cases. There was no bony involvement in any of the cases. Nearly $75 \%$ of these masses crossed the midline [Figure 3]. Histological confirmation of Wilms' tumor was obtained in all cases.

\section{DISCUSSION}

Imaging plays a crucial role in early diagnosis of Wilms' tumor, to distinguish it from all other causes of abdominal distention. Previous studies in this institution delved on preoperative imaging with ultrasonography and intravenous urography (IVU) for its diagnosis. ${ }^{[7]}$ The average age of children with Wilms' tumor in this study is similar those found in the literature. ${ }^{[3]}$

Conventional ultrasound is the most common method for initial diagnosis of Wilms' tumor due its non-invasiveness,

\begin{tabular}{lcc}
\hline Table 1: CT scan findings & & \\
\hline Findings & Frequency & Percentage \% \\
Abdominal distension & 12 & 100 \\
Soft tissue mass & 12 & 100 \\
Bulging of the flanks & 12 & 100 \\
Loss of Psoas line & 12 & 100 \\
Right sided tumour & 5 & 42 \\
Left sided tumour & 4 & 33.3 \\
Bilateral tumour & 3 & 25 \\
Tumour crossing the midline & 9 & 75 \\
Calcification & 2 & 17 \\
Heterogenous contrast enhancement & 12 & 100 \\
Vascular involvement & 3 & 25 \\
Lymph Node enlargement & 6 & 50 \\
Bony involvement & $\mathrm{Nil}$ & 0 \\
\hline
\end{tabular}

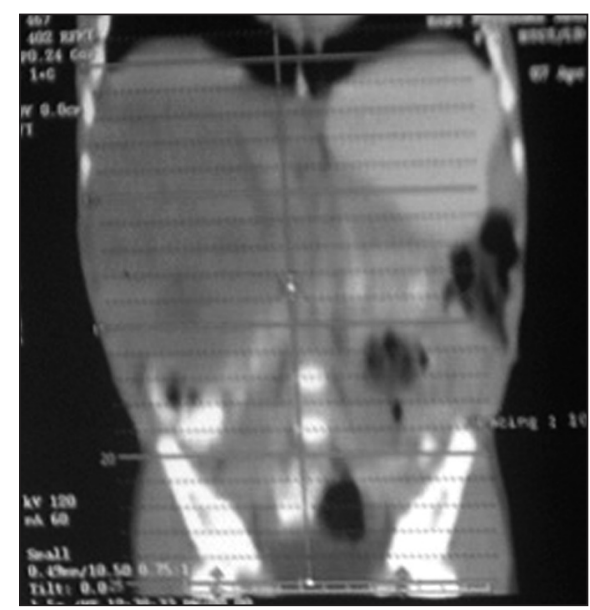

Figure 1: Computed tomography scanogram showing bulging of the flanks, more on the right side and a large right hypodense mass causing loss of the psoas muscle outline and extending from below the right hemidiaphragm to the right iliac crest. Bowel loops are displaced downwards and to the right. The mass is seen to cross the midline

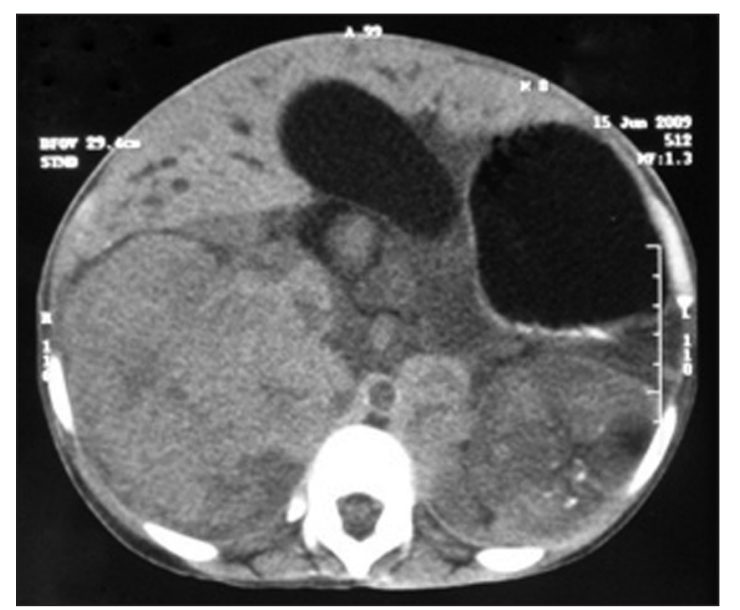

Figure 2: Axial computed tomography slice showing discrete, punctuate calcifications within a well-defined left renal mass in a case of bilateral Wilms' tumour. The paraortic lymph node are involved and the right renal mass is seen breaking through the renal capsule antero-medially

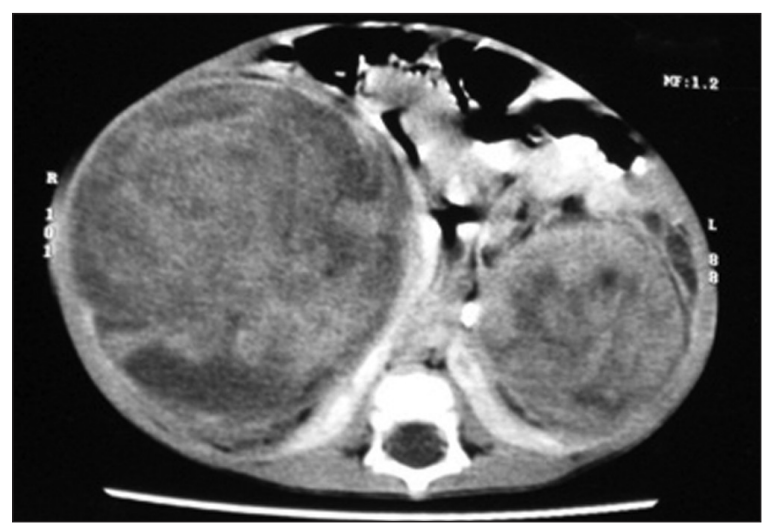

Figure 3: Contrast enhanced axial computed tomography scan showing bilateral Wilms' tumour with fullness of both flanks and the right renal mass is seen crossing the midline. Heterogenous contrast enhancement is noted. The bowel loops are displaced medially and sandwitched the two renal masses 
affordability and availability. ${ }^{[12]}$ However, it is operator dependent and has poor cross-sectional anatomical information. An important draw back to its use is that, it is less accurate in tumor staging, which is required for the effective treatment of this malignancy. CT scan however, helps staging of the tumor. IVU can assess physiological or functional ability of the diseased kidneys and also studies have shown that IVU was helpful in pre-operative differentiation between neuroblastoma and Wilms' tumor as it gives supplementary information on the size and extent of the solid nature of the tumor. ${ }^{[13]}$ However, IVU was inferior to CT in differentiating between solid tumors and benign lesions such as cysts and hydronephrosis. ${ }^{[13]}$ Scott et al. ${ }^{[14]}$ stated that tumors that crossed the midline were suggestive of neuroblastomas rather than Wilms' tumor and is better evaluated and confirmed with CT than IVU. Contrary to this claim but in tandem with the findings of Scott et al. ${ }^{[14]}$ in their study, the present study revealed that majority of the tumors crossed the midline. This can be attributed to the delay in the diagnosis as a result of patients presenting late to the hospital. Cost-effective radiation dose to patients is another advantage of CT over IVU. ${ }^{[14]}$

Modern radiological imaging methods have superseded conventional ultrasound and IVU in the pre-operative evaluation of patients with Wilms' tumor. Modern techniques such as CT and MRI have been found to be more accurate than ultrasonography in tumor staging of Wilms' tumor ${ }^{[12,15]}$ while chest and abdominal CT may be performed during the examination to determine the presence or absence of pulmonary metastases. ${ }^{[15]}$

CT is currently the technique of choice in the diagnosis and staging of renal masses in children, and is particularly relevant in recognition of size and site of lesion and densitometric patterns. ${ }^{[2]}$ It also provides an excellent visualisation of contiguous structures like vessels and lymph nodes. ${ }^{[2]}$ All these were confirmed in this study.

On CT, Wilms' tumor usually appears as a bulky, spherical intrarenal mass, very often with a well-defined rim of compressed renal parenchyma or pseudocapsule surrounding it [Figure 3], as was corroborated by this study. Some tumors that arise from the periphery of the cortex may grow in an exophytic manner with bulk of the tumor seen outside the renal cortex. ${ }^{[15]}$ In general, a heterogenous mass replacing the kidney and displacing adjacent organs can be demonstrated. The tumor is hypodense compared to the normal renal parenchyma on contrast enhanced CT scans with the areas of low attenuation coinciding with tumor necrosis, fat deposition or both. ${ }^{[16]}$ All patients in this study also demonstrated these features. More of the patients in this study showed calcifications in the mass when compared to the study by
Fishman et al..$^{[15]}$ and Geller et al. ${ }^{[16]}$ where only about $13 \%$ of Wilms' tumor presented with calcifications on nonenhanced scans. About $10 \%$ of patients exhibit poor or absent renal function of the involved kidney because of venous tumor extension, compression of the collecting system or extensive tumor infiltration throughout the kidney. ${ }^{[17]}$ Only $25 \%$ of our patients demonstrated vascular encasement in the present study.

Kim and Chung, ${ }^{[3]}$ Dome and Huff ${ }^{[5]}$ claim that synchronous bilateral disease occurs in about $5-8 \%$ of children, affecting more girls. This is much less than this study revealed, as $25 \%$ of the infants in the present study had bilateral Wilms disease and incidentally, they were all boys.

Intracaval extension of the tumor can be defined on CT scan or ultrasound. ${ }^{[3]}$ CT scan has been shown to be an accurate means of diagnosing, staging and monitoring treatment. ${ }^{[4]}$

Recently, MRI was claimed to be the imaging modality of choice to diagnose Wilms tumor, suggesting that it is superior to CT and to ultrasound in defining the extent of intravascular involvement. ${ }^{[3]}$

Wilms' tumors are staged on the basis of anatomic involvement of the tumor. ${ }^{[3]}$

CT is also useful for identification of lymph node enlargement, which could either be tumor metastases or reactive changes as it is impossible to confirm the former from CT. ${ }^{[12,18]}$ In half of the study subjects, the lymph nodes were involved, which therefore supports the fact that most of the patients in this environment present late. It is therefore pertinent to mention here that because our state Government offers treatment to pediatric patients almost at no cost, increasing the awareness of parents could help catch these children earlier and thus reduce the morbidity and mortality from this disease entity.

The inferior vena cava is involved by tumor thrombus in $4-10 \%$ of Wilms tumor. ${ }^{[15]}$ This is contrary to our findings where vascular involvement was $17 \%$. This could also be attributed to the late presentation of patients.

In our prospective study of 12 patients, the scanogram showed flank bulge in all 12 patients (100\%) [Figure 1], which supports the bulky size of these tumors because of late presentation in our environment. Most of these tumors crossed the midline, contrary to previous studies ${ }^{[14]}$ [Figure 3].

Our findings correlated with intraoperative findings and histological diagnosis, thus buttressing the claim that CT scan is superior to IVU and ultrasonography. 
One of the limitations of this study was the unavailability of spiral CT scan in our center. It has been shown that spiral CT further improves the quality of examination, has the ability for faster image acquisition thus reducing the period of sedation, consequently lowering the radiation dose ${ }^{[2]}$ in keeping with the "Image gently" slogan in pediatric imaging. Spiral CT would also improve the enhancement of the lesion and abdominal vessels. The latter are better studied using multi-planar reconstructions.

Furthermore, the non-inclusion of a chest CT with the abdominal CT is a limitation as this would have detected lung metastases, which is a determinant of the treatment option.

The various findings from this study, correlated with the operative and histopathology results.

\section{CONCLUSION}

Based on the limitation of this study it can be concluded that we have demonstrated that most of the cases of Wilms' tumor in this environment cross the midline, a quarter of them were bilateral (more in boys) and that more of them demonstrated calcification contrary to what was seen in the literature. Lymph nodes were involved and vessels were encased because they present late. Abdominal CT scan, in spite of its high radiation dose is relevant and accurate in making a diagnosis of Wilms' tumor and is important for its early and accurate management in resource poor settings where patients usually present late for treatment.

\section{REFERENCES}

1. Ekenze SO, Heij HA, Youngson GG. Kidney tumours. Paeditric Surgery: A Comprehensive Text for Africa. Seattle, WA: Global HELP Organisation; 2011. p. 610-3.

2. Miele V, Galluzzo M, Bellussi A, Valenti M. Spiral computerized tomography in the study of renal neoplasms in children. Radiol Med 1998;95:486-92.
3. Kim S, Chung DH. Pediatric solid malignancies: Neuroblastoma and wilms tumor. Surg Clin North Am 2006;86:469-87.

4. Lowe RE, Cohen MD. Computed tomographic evaluation of Wilm's tumour and neuroblastoma. Radiographics 1984;4:915-28.

5. Dome JS, Huff V. In: Pagon RA, Adam MP, Bird T, Dolan CR, Fong CT, Smith RJH, Stephens K. editors. GeneReviews ${ }^{\circledR}$ [Internet]. Seatke (WA), University of Washington, Seattle, 1993-2014. 2003. Last accessed on 2013 September.

6. Riccabona M. Imaging of renal tumours in infancy and childhood. Eur Radiol 2003;13 Suppl 4:L116-29.

7. Osuoji RI, Williams OM, Ajai OT, Idika OC, Abolarinwa AA Bankole MA. Wilms' tumour: Experience in a developing tertiary centre in Nigeria. East Cent Afr J Surg 2011;16:52-8.

8. Rohrschneider WK, Weirich A, Rieden K, Darge K, Tröger J, Graf N. US, CT and MR imaging characteristics of nephroblastomatosis. Pediatr Radiol 1998;28:435-43.

9. Miniati D, Gay AN, Parks KV, Naik-Mathuria BJ, Hicks J, Nuchtern JG, et al. Imaging accuracy and incidence of Wilms' and nonWilms' renal tumors in children. J Pediatr Surg 2008;43:1301-7.

10. Khan AN, Lin EC. Available from: http://emedicine.medscape. com/article/415012-overview. Last accessed on 2013 July.

11. Jone $\mathrm{J}$ and Gaillard F. Neuroblastoma vs Wilms tumour. Available from: http://www.radiopedia.org/articles/neuroblastoma-vswilms-tumour-1. Last accessed on 2013 November.

12. Cushing B, Slovis TL. Imaging of Wilms' tumor: What is important! Urol Radiol 1992;14:241-51.

13. Damgaard-Pedersen K. CT and IVU in the diagnosis of Wilms' tumour. A comparative study. Pediatr Radiol 1980;9:207-11.

14. Scott DJ, Wallace WH, Hendry GM. With advances in medical imaging can the radiologist reliably diagnose Wilms' tumours? Clin Radiol 1999;54:321-7.

15. Fishman EK, Hartman DS, Goldman SM, Siegelman SS. The CT appearance of Wilms tumor. J Comput Assist Tomogr 1983;7:659-65.

16. Geller E, Smergel EM, Lowry PA. Renal neoplasms of childhood. Radiol Clin North Am 1997;35:1391-413.

17. Shneta B, Vikram SD. Kidney. In: Haaga JR, Vicram SD, Forsting M, Gilkenson RC, Hyun $\mathrm{KH}$, Sundaram M, editors. CT and MRI of Whole Body. $5^{\text {th }}$ ed., Vol. 2. China: Mosby Elsevier; 2008. p. 1902.

18. Siegel MJ. MR imaging of pediatric abdominal neoplasms Magn Reson Imaging Clin N Am 2000;8:837-51.

How to cite this article: Olukayode AA, Richard IO, Rachael AA, Babajide OB, Ireti OF, Gbolahan OA. Pattern of computed tomography scan findings in children with Wilms' tumor in a tertiary hospital in Lagos, Nigeria. Indian J Med Paediatr Oncol 2014;35:31-5.

Source of Support: Nil. Conflict of Interest: None declared.

Announcement

\section{iPhone App}

Download

iPhone, iPad application
A free application to browse and search the journal's content is now available for iPhone/iPad. The application provides "Table of Contents" of the latest issues, which are stored on the device for future offline browsing. Internet connection is required to access the back issues and search facility. The application is Compatible with iPhone, iPod touch, and iPad and Requires iOS 3.1 or later. The application can be downloaded from http://itunes.apple.com/us/app/medknow-journals/ id 458064375 ? $\mathrm{s}=1 \& \mathrm{mt}=8$. For suggestions and comments do write back to us. 